\title{
Ultrasound monitoring of the treatment of clinically significant knee osteoarthritis
}

\author{
Vojtassak Jr $\mathbf{J}^{1}$, Vojtassak Sen $\mathbf{J}^{1,2}$ \\ Orthos Paidion, sro, Bratislava, Slovakia. j.vojtassak@gmail.com
}

\begin{abstract}
Objectives: The study presented an ultrasound (US) monitoring of treatment as a new imaging US method with the results of therapy of clinically significant knee osteoarthritis.

Background: X-ray is widely used for knee osteoarthritis classification, which does not involve the evaluation of the soft tissue. High frequency and high resolution US of joints (arthrosonography, echoarthrography) assess not only morphologic but also functional changes in the knee joint.

Methods: In the prospective study, 110 patients with clinically significant knee osteoarthritis were treated nonoperative. US examination and US monitoring of therapy was performed during 24 weeks therapy period. A remission of pathomorphologic (marginal osteofytes) and pathophysiologic (effusion in anterior knee and Baker's cyst) attributes were evaluated according the US classification.

Results: Pathomorphologic attributes changes showed a static state, without remission or progression. Pathophysiologic attributes changes showed a remission during the study period. The highest remission was in the first three weeks, $60 \%$ anterior knee effusion and $62 \%$ Baker's cyst. At the end of study, no changes from the initial US grade was observed in $16 \%$ of effusion in anterior knee and $22 \%$ of Baker's cyst. Therapeutic resistant Baker's cyst was present at the end of study in $36 \%$.

Conclusion: We demonstrated a new method - US monitoring of therapy, which can objectivize the efficiency of treatment of clinically significant knee osteoarthritis. We would recommend US monitoring of therapy for the routine use in orthopedic clinical praxis (Tab. 6, Graph 3, Fig. 3, Ref. 15). Text in PDF www.elis.sk.

Key words: clinically significant knee osteoarthritis, ultrasound monitoring of therapy, arthrosonography, echoarthrography.
\end{abstract}

Clinically significant knee osteoarthritis is one of the clinical forms of the knee osteoarthritis with clinical manifestation and functional disorders, which can be regularly visualized by X-ray, computed tomography (CT) or magnetic resonance imaging (MR) (1). These imaging techniques help us to evaluate and classify degenerative changes of osteoarthritis. X-ray is widely used for knee osteoarthritis classification, which does not involve the evaluation of soft tissue of the knee.

Ultrasound (US) is a relatively newer imaging technique in musculoskeletal system dominantly for diagnostics, navigated interventions and monitoring of treatment (2). High frequency and high resolution US of joints (arthrosonography, echoarthrography) assess not only morphologic but also functional changes in the knee joint. Static and dynamic evaluations are big advantages of this imaging technique (3).

The complex anterior and posterior quantitative US evaluation and monitoring of therapy of clinically significant knee osteoarthritis was not published jet and is not generally used in orthopaedic praxis.

${ }^{1}$ Orthos Paidion, sro, Bratislava, Slovakia, and ${ }^{2}$ Department of Orthopaedics, Faculty of Medicine, Slovak Medical University in Bratislava, Bratislava, Slovakia

Address for correspondence: J. Vojtassak Jr, MD, Orthos Paidion, sro, Vajnorska 40, SK-832 63 Bratislava, Slovakia.

Phone: +421.907220093
The aim of this article was to present US monitoring of treatment as a new imaging US method with the results of the therapy of clinically significant knee osteoarthritis in clinical praxis.

\section{Methods}

In this prospective study, 110 patients from 1.5.2008 to 30.4.2012 with clinically significant knee osteoarthritis were examined and treated non-operative in orthopedic praxis. Patients, who had surgery, were excluded from this study.

All patients were physically examined and X-ray confirmed diagnosis. MR or CT was done in some cases. US examination and US monitoring of therapy was performed during the therapy period.

Patients were from 30 to 90 years old, the average age was 55 . Sex distribution was $58 \%$ female and $42 \%$ male.

The complex therapy of clinically significant knee osteoarthritis covered US navigated intervention (joint punction or arthrocentesis, intraarticular aplication of anti-inflammatory agents, hyaluronic acid injections) oral intake of SYRADOA (symptomatic rapid acting drugs of osteoarthritis) and SYSADOA (symptomatic slow acting drugs of osteoarthritis), adjustment of physical activity, physiotherapy and a reduction of high body mass index.

A remission or progression of knee osteoarthritis and its decompensated form was evaluated by US classification, which we use in clinical praxis. US evaluation was divided in morphology 
Tab. 1. US classification of pathomorphologic attributes represented measurements of marginal osteofytes on dominant or more affected compartment of the knee joint.

\begin{tabular}{ll}
\hline grade 0 & without ostefytes \\
\hline grade I & osteofytes $0-0.9 \mathrm{~mm}$ \\
\hline grade II & osteofytes $1.0-2.9 \mathrm{~mm}$ \\
\hline grade III & osteofytes $3.0-4.9 \mathrm{~mm}$ \\
\hline grade IV & osteofytes $5.0 \mathrm{~mm}$ and more \\
\hline
\end{tabular}

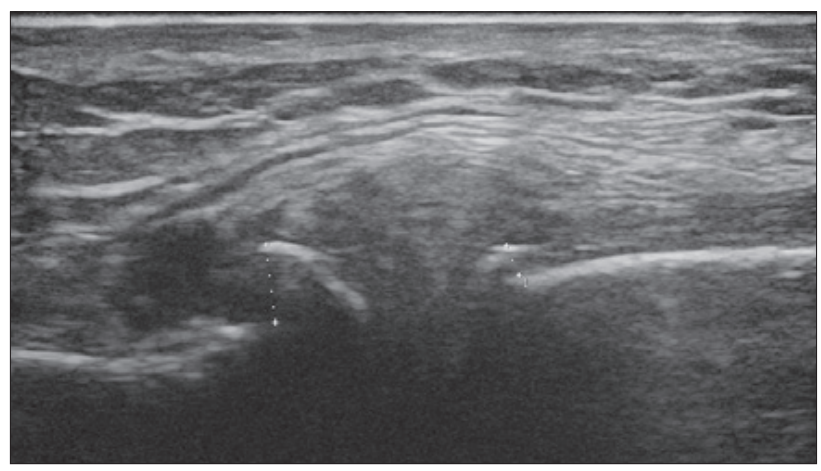

Fig. 1. US measurements of marginal osteofytes on dominant or more affected compartment of the knee joint.

(pathomorphologic) attributes and functional (pathophysiologic) attributes.

Pathomorphologic attributes classification (Tab. 1) covered the US measurements of marginal osteofytes on dominant or more affected compartment of the knee joint (Fig. 1). Measurements were done in the long axis of the knee perpendicular to joint. We recorded quantitatively the biggest value of marginal osteofytes from measurements. Dominant compartments were in

Tab. 2. US classification of pathophysiologic attributes represented measurements of effusions height in anterior part of affected knee in suprapatellar recess.

\begin{tabular}{ll}
\hline grade 0 & without effusion \\
\hline grade I & $\begin{array}{l}\text { Effusion to } 1.9 \mathrm{~mm} \text { only during mus. quadriceps con- } \\
\text { traction by patient or during pressure on medial or lat- } \\
\text { eral parapatellar recess by physician }\end{array}$ \\
\hline grade II & Effusion $2.0-2.9 \mathrm{~mm}$ \\
\hline grade III & Effusion $3.0-9.9 \mathrm{~mm}$ \\
\hline grade IV & Effusion $10.0 \mathrm{~mm}$ and more \\
\hline qualitatively & $\begin{array}{l}\text { anechoic/hypoechoic } \\
\text { anechoic/hypoechoic with fibrous tissue } \\
\text { homogenous echogenicity } \\
\text { heterogeneous echogenicity }\end{array}$
\end{tabular}

Tab. 3. US classification of pathophysiologic attributes represented measurements of effusions in posterior part (Baker's cyst) of affected knee.

\begin{tabular}{ll}
\hline grade 0 & without effusion or physiologic Baker's cyst \\
\hline grade I & Effusion till 9.9 mm \\
\hline grade II & Effusion $10.0-29.9 \mathrm{~mm}$ \\
\hline grade III & Effusion $30.0-49.9 \mathrm{~mm}$ \\
\hline grade IV & Effusion $50.0 \mathrm{~mm}$ and more \\
\hline qualitatively & $\begin{array}{l}\text { anechoic/hypoechoic } \\
\text { anechoic/hypoechoic with fibrous tissue or septum } \\
\text { homogenous echogenicity } \\
\text { with lose body inside Baker's cyst }\end{array}$
\end{tabular}

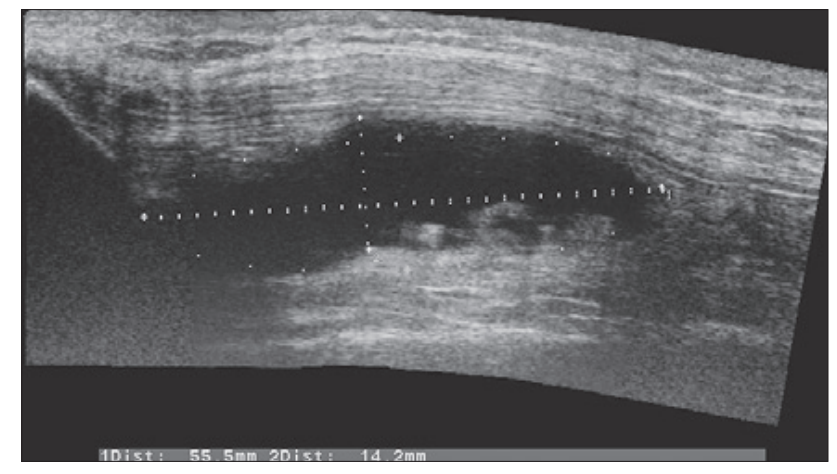

Fig. 2. In the anterior part of the knee, the examination was done is suprapatellar recess.

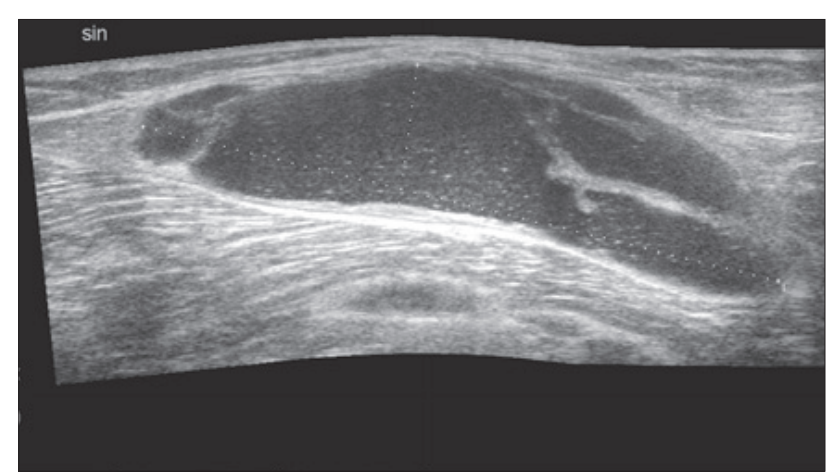

Fig. 3. In the posterior part of the knee, Baker's cyst was examined and measured.

the following sequence: medial, lateral, femoropatellar and multicompartment.

Pathophysiologic attributes classification covered US measurements of effusions height in the anterior (Tab. 2) and the posterior (Tab. 3) part of affected knee. In the anterior part of the knee, the examination was done is suprapatellar recess (Fig. 2). In the posterior part of the knee, Baker's cyst was examined and measured (Fig. 3). Measurements were done in the long axis of joint in anterior part of the knee, in the long and short axis in the posterior part of the knee. We recorded quantitatively the biggest value of effusions height and recorded qualitatively the character of effusion. We considered Baker's cyst grade 0 and grade I as clinically acceptable and gr II, III and IV as therapeutically resistant.

Pathomorphologic and pathophysiologic attributes were analyzed separately. Patients were divided into 8 groups before therapy in the week 0 according to US classification of pathophysiologic changes. Group A represented effusion grade IV, group B effusion grade III, group $\mathrm{C}$ effusion grade II, group D effusion grade I. Group E represented Baker's cyst grade IV, group F Baker's cyst grade III, group G Baker's cyst grade II, group H Baker's cyst grade I. Patients were examined in the week 0, 3, 6, 16, 24 and treatment was aplied in the week $0,3,6,16$. A progression or remission of each group was recorded and analyzed by US classification. We considered the remission when the number of patients decreased in the initial grade (week 0 ) in every group. We analyzed the remission between the weeks $0-3,3-6,6-16$, 
Tab. 4. Distribution of pathomorphologic attributes changes of the knee according to US classification and weeks.

\begin{tabular}{lccccc}
\hline Marginal osteofytes & week 0 & week 3 & week 6 & week 16 & week 24 \\
\hline grade I & 14 & 14 & 14 & 14 & 14 \\
grade II & 29 & 29 & 29 & 29 & 29 \\
grade III & 38 & 38 & 38 & 38 & 38 \\
grade IV & 29 & 29 & 29 & 29 & 29 \\
\hline
\end{tabular}

Tab. 5. Distribution of pathophysiologic attributes changes - effusion in anterior knee according to US classification and weeks.

\begin{tabular}{|c|c|c|c|c|c|c|c|c|c|c|c|c|c|c|}
\hline \multirow[b]{2}{*}{ grade } & \multicolumn{5}{|c|}{ group A } & \multicolumn{4}{|c|}{ group B } & \multicolumn{3}{|c|}{ group C } & \multicolumn{2}{|c|}{ group D } \\
\hline & IV & III & II & I & 0 & III & II & I & 0 & II & I & 0 & I & 0 \\
\hline week 0 & 20 & - & - & - & & 52 & - & - & - & 26 & - & & 12 & - \\
\hline k 3 & 7 & 8 & 3 & 2 & 0 & 16 & 22 & 13 & 1 & 9 & 14 & & 7 & 5 \\
\hline k 6 & 2 & 3 & 12 & 3 & 0 & 12 & 18 & 20 & 2 & 5 & 15 & 6 & 3 & 9 \\
\hline 16 & 2 & 3 & 12 & 3 & 0 & 11 & 18 & 21 & 2 & 4 & 14 & 8 & 2 & 10 \\
\hline veek 24 & 2 & 4 & 11 & 3 & 0 & 11 & 22 & 19 & 0 & 4 & 17 & 5 & 1 & 11 \\
\hline
\end{tabular}

Tab. 6. Distribution of pathophysiologic attributes changes - Baker's cyst according to US classification and weeks.

\begin{tabular}{|c|c|c|c|c|c|c|c|c|c|c|c|c|c|c|}
\hline \multirow[b]{2}{*}{ grade } & \multicolumn{5}{|c|}{ group $\mathrm{E}$} & \multicolumn{4}{|c|}{ group F } & \multicolumn{3}{|c|}{ group $\mathrm{G}$} & \multicolumn{2}{|c|}{ group $\mathrm{H}$} \\
\hline & IV & III & II & I & 0 & III & II & I & 0 & II & I & 0 & I & 0 \\
\hline week 0 & 17 & - & - & - & - & 15 & - & - & -7 & 10 & - & - & 4 & - \\
\hline ek 3 & 5 & 6 & 4 & 2 & 0 & 5 & 5 & 4 & 1 & 4 & 4 & 2 & 2 & 2 \\
\hline eek 6 & 3 & 5 & 6 & 3 & 0 & 5 & 4 & 4 & 2 & 3 & 5 & 2 & 1 & 3 \\
\hline veek 16 & 3 & 4 & 6 & 4 & 0 & 4 & 6 & 4 & 1 & 2 & 5 & 5 & 1 & 3 \\
\hline week 24 & 3 & 5 & 7 & 2 & 0 & 4 & 7 & 4 & 0 & 2 & 5 & 3 & 1 & 3 \\
\hline
\end{tabular}

16-24 and 0-24. In the Baker's cyst, we analyzed the remission according to US classification and the remission of total number of therapeutic resistant Baker's cyst.

The selection of the aplied treatment depended on the grade of US classification of pathomorphologic changes of the knee. If grade II or more in the week $0,3,6,16$ was present, US navigated intervention was preferred.

US examination and monitoring was done using the ultrasound system Aloka Prosound Alpha7 and F75 (Hitachi Aloka Medical, Ltd., Japan) with multifrequency and high frequency linear probe (5-13 MHz).

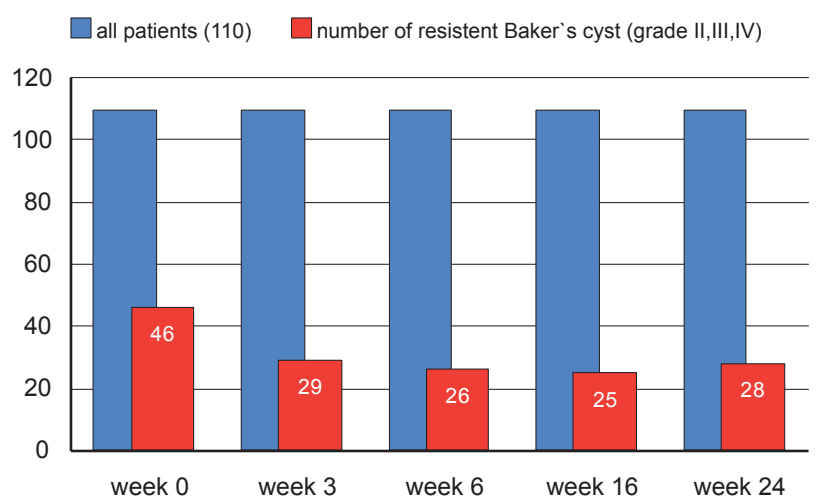

Graph 1.Decreasing number of resistant Baker's cyst (grade II,III,IV) during therapy.

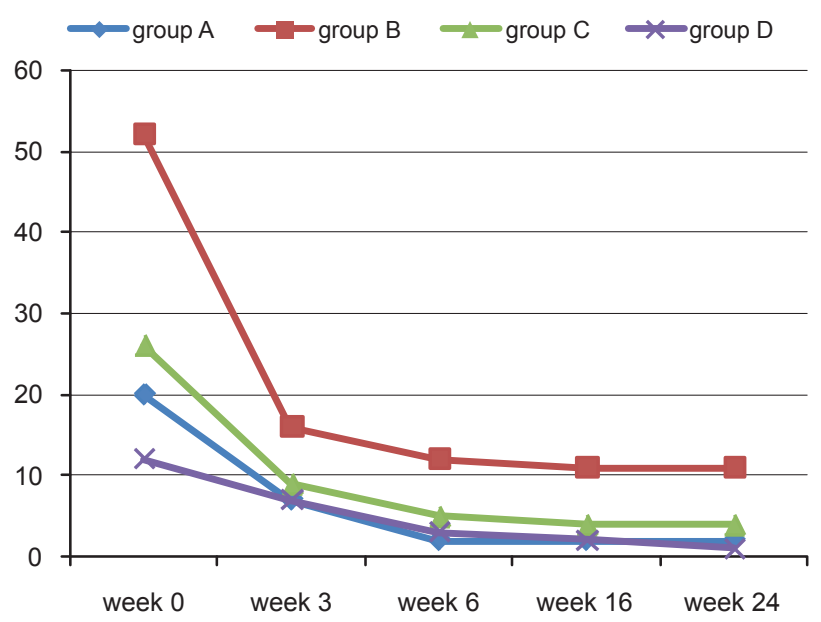

Graph 2 .Decreasing numbers of patients in each group in the same grade during therapy. Group A in grade IV, group B in grade III, group C in grade II, group D in grade I during therapy).

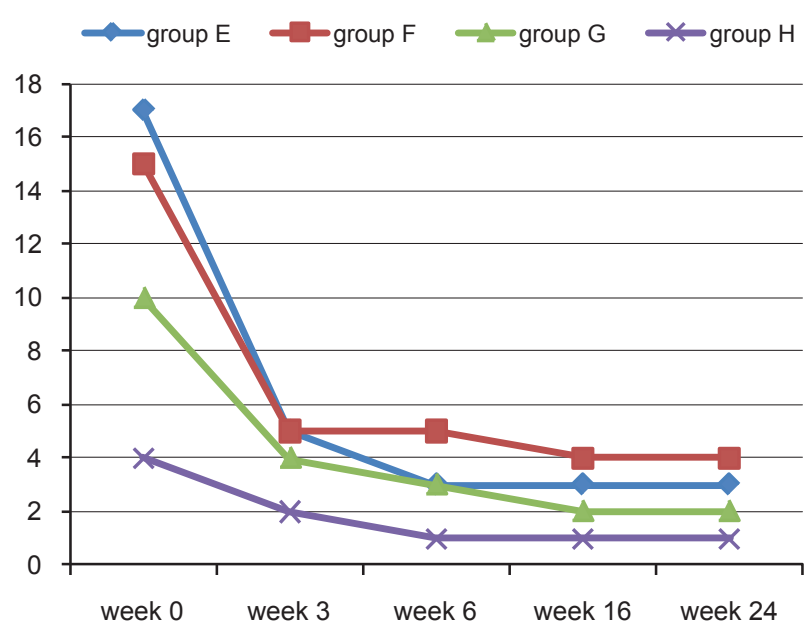

Graph 3.Decreasing numbers of patients in each group in the same grade during therapy. Group E in grade IV, group F in grade III, group $\mathrm{G}$ in grade $\mathrm{II}$, group $\mathrm{H}$ in grade $\mathrm{I}$ during therapy.

\section{Results}

Pathomorphologic attributes changes (marginal osteofytes) evaluated by US did not show any progress during the study period (Tab. 4.).

Pathophysiologic attributes changes (effusion in anterior knee, Baker's cyst) did show US remission during the study period (Tabs 5 and 6).

A decreasing total number of therapeutically resistant Baker's cyst (grade II, III, IV) (Graph 1) was the highest in the week 0-3 in $37 \%$ (17 patient), in the week 3-6 it was $10 \%$ (3 patients), in the week 6-16 it was $4 \%$ (1 patient), in the week 16-24 it was $12 \%$ (-3 patients), during the whole therapy period (week $0-24$ ) in $39 \%$ (18 patients). An increasing total number ( +3 patients) of resistant Baker's cyst in the week 16-24 was due to the group E grade II,I II and the group F grade II. Therapeutic resistant Baker's cyst was 
present at the end of the study (week 24) in $36 \%$ (28 patients) in comparison with the initial state (week 0 ) in $42 \%$ (46 patients).

US remission of effusion in the anterior knee (Graph 2) was the highest confirmed in the week $0-3$ in $60 \%$ patients ( 13 patients in the group A in grade IV, 36 patients in the group B in grade III, 17 patients in the group C in grade II, 5 patients in the group D in grade I), in week 3-6 in 50\% patients (5 patients in the group A in grade IV, 4 patients in the group B in grade III, 4 patients in the group $\mathrm{C}$ in grade II, 4 patients in the group D in grade I), in the week $6-16$ in $15 \%$ patients ( 0 patients in the group A in grade IV, 1 patient in the group B in grade III, 1 patient in the group C in grade II, 1 patient in the group D in grade I), in the week 16-24 in $13 \%$ patients $(0$ patients in the group $\mathrm{A}$ in grade IV, 0 patients in the group B in grade III, 0 patients in the group C in grade II, 1 patient in the group D in grade I). At the end of study (week 24), $16 \%$ (18 patients) did not change the initial US grade of effusion in the anterior knee.

US remission of Baker's cyst (Graph 3) was the highest confirmed in the week $0-3$ in $62 \%$ patients (12 patients in the group $\mathrm{E}$ in grade IV, 10 patients in the group F in grade III, 6 patients in the group $\mathrm{G}$ in grade II, 2 patients in the group $\mathrm{H}$ in grade I), in the week $3-6$ in $29 \%$ patients ( 2 patients in the group $\mathrm{E}$ in grade IV, 0 patients in the group $F$ in grade III, 1 patient in the group $G$ in grade II, 1 patient in the group $\mathrm{H}$ in grade I), in the week 6-16 in $13 \%$ patients ( 0 patients in the group $\mathrm{E}$ in grade IV, 1 patient in the group F in grade III, 1 patient in the group $\mathrm{G}$ in grade II, 0 patients in the group $\mathrm{H}$ in grade I), in the week 16-24 in $0 \%$ patients ( 0 patients in the group $\mathrm{E}$ in grade IV, 0 patients in the group $\mathrm{F}$ in grade III, 0 patients in the group $\mathrm{G}$ in grade II, 0 patients in the group $\mathrm{H}$ in grade I). At the end of study (week 24), $22 \%$ (28 patients) did not change the initial US grade of Baker's cyst.

During US monitoring of the therapy, we did not notice any complication. All patients knees were good visualized and the values were reproducible.

\section{Discussion}

Knee osteoarthritis is a degenerative joint disease with a dominant affected cartilage. Knee osteoarthritis is considered as an irreversible joint diseases because of limited repairing capability of cartilage. Good treatment result is considered when arthritic process is stoped or slowed down (4).

Repairing capability and metabolism of cartilage depends on dynamic equilibrium between formation and breakdown of the cartilaginous matrix, which is regulated by an interplay of anabolic influences (e.g., insulin-like growth factors [IGF] I and II) and catabolic influences (e.g., interleukin-1, tumor necrosis factor [TNF] alpha, and proteinases) (1).

Clinical symptoms of knee osteoarthritis depend on static and biomechanical attributes of joint, which are in arthritis joint reduced. Decompensated gonarthrosis is synonym for a clinically significant knee osteoarthritis common used in our region.

Symptoms of clinically significant knee osteoarthritis develop when an inequilibrium between the aplied static and dynamic load on the knee and capability of the knee is present. Symptoms and functional disorders of clinically significant knee osteoarthritis are pain, swelling, joint effusion, synovitis, loss of range of motion, axial deviation of join, muscle hypotrophy, instability, impaired walking stereotype (4).

Major symptoms of clinically significant knee osteoarthritis, which tend the patients to visit orthopaedic praxis, are pain and synovitis with effusion (4). An association between the US finding of suprapatellar effusion and medial compartment synovitis with a significantly associated knee pain was confirmed (5). Joint synovitis with effusion is best evaluated and quantificated in the suprapatellar recess, less in the parapatellar medial or lateral recess of the knee.

Baker's cyst is another specific sign of clinically significant knee osteoarthritis (6). Longstanding fluid collection in the anterior knee tends to progressive fill the communicating bursitis between the medial gastrocnemius and semimembranosus tendon what leads to the formation of Baker's cyst. Baker's cyst is also common in the rheumatoid arthritis (3).

The treatment of clinically significant knee osteoarthritis involves intra-articular anti-inflammatory agents, corticosteroid, hyaluronic acid injections, oral intake of SYRADOA (symptomatic rapid acting drugs of osteoarthritis) and SYSADOA (symptomatic slow acting drugs of osteoarthritis), decreased physical activity, physiotherapy and reduction of high body mass index. Discussion of intra-articular corticosteroid injection is still controversial, but many authors reported good results with a low side effect especially when aplied under US navigation with exact focusing and placing in target tissue (7-9).

The physical examination is mandatory in all orthopaedic examination (10). Regularly used diagnostic imaging techniques are $\mathrm{X}$-ray, CT, MR, occasionally scintigraphy, and in certain causes arthroscopy. In spite of good clinical data and efficiency, these imaging techniques cannot be used for routine and frequent use in the same patient. We tried to find out suitable imaging techniques for everyday use in orthopaedic praxis eligible for diagnostics and monitoring of treatment. US fulfilled our concept of radiation-less, not biologically invasive, well-tolerated by patients, repetitive and good reproducible imagining techniques for clinically significant knee osteoarthritis (11). US with high spatial resolution $(0,1 \mathrm{~mm})$ for morphologic and functional changes is suitable for quantification, which cannot be assessed by everyday physical examination (12). Superficial soft tissues are best visualized by US. Although US has limitation in whole bone visualization, superficial bone structures as bone surface, cartilage surface and cortical layer are well visualized $(13,14)$.

In clinical praxis, Kellgren-Lawrence X-ray Classification (4) is widely used for knee osteoarthritis. This classification omits the evaluation of soft tissue, which play an important role in the decompensated form of knee osteoarthritis (5). A remission of decompensated form of knee osteoarthritis is in clinical praxis evaluated qualitatively by palpation of synovitis with effusion in the knee. US enables to visualize and quantify the remission of synovitis with effusion during monitoring of therapy. Some authors (15) described US finding $55 \%$ suprapatellar effusion and $25 \%$ Baker's cyst on symptomatic knees 
and $22 \%$ suprapatellar effusion and $5 \%$ the Baker's cyst on asymptomatic knees.

We did not use US as a single imaging technique, but we used it as an additional imaging technique for musculoskeletal system. Nowadays, we did not find only one ideal imaging technique for soft tissues and bones suitable for diagnostics and monitoring of treatment. We prefer US for diagnostics and monitoring of treatment of decompensated knee osteoarthritis in spite of disadvantage and limitation of US.

In conclusion, we demonstrated in this study a new method - US monitoring of therapy, which can objectivize the efficiency of the treatment of clinically significant knee osteoarthritis. We assessed dynamics of pathomorphologic attributes (marginal osteofytes) and pathophysiologic attributes (effusion in anterior knee and Baker's cyst) in 24 weeks during therapy. Pathomorphologic attribute changes showed a static state, without the remission or progression. Pathophysiologic attribute changes showed the remission during the study period. The highest remission was in the first three weeks $60 \%$ anterior knee effusion and $62 \%$ Baker's cyst, $50 \%$ decreasing of total number of Baker's cyst. At the end of study (week 24), $16 \%$ (18 patients) did not change the initial US grade of effusion in the anterior knee, $22 \%$ (28 patients) did not change the initial US grade of Baker's cyst. Therapeutically resistant Baker's cyst was present at the end of the study (week 24 ) in $36 \%$ (28 patients). We would recommend US monitoring of the therapy for routine use in orthopedic clinical praxis.

\section{References}

1. Michael JW, Schlüter-Brust KU, Eysel P. The epidemiology, etiology, diagnosis and treatment of osteoarthritis of the knee. Dtsch Arztebl Int 2010; 107 (9): 152-162.

2. Royall NA, Farrin E, Bahner DP, Stawicki SP. Ultrasound-assisted musculoskeletal procedures: A practical overview of current literature, World J Orthop 2011; 2 (7): 57-66.

3. Martino F, Silvestri E, Grassi W, Garlaschi G: Musculoskeletal Sonography: technique, anatomy, semeiotics and pathological findings in rheumatic diseases. Milan Berlin Heidelberg New York; Springer, 2007, $1-207$.
4. Vojtaššák J: Ortopédia a traumatológia. Bratislava; SAP, 2006, 1-577.

5. Wu PT, Shao CJ, Wu KC, Wu TT, Chern TC, Kuo LC, Jou IM. Pain in patients with equal radiographic grades of osteoarthritis in both knees: the value of gray scale ultrasound. Osteoarthritis Cartilage 2012; 20 (12): 1507-1513.

6. Bandinelli F, Fedi R, Generini S et al. Longitudinal ultrasound and clinical follow-up of Baker's cysts injection with steroids in knee osteoarthritis. Clin Rheumatol 2012; 31 (4): 727-731.

7. Di Sante L, Paoloni M, Dimaggio M. Ultrasound-guided aspiration and corticosteroid injection compared to horizontal therapy for treatment of knee osteoarthritis complicated with Baker's cyst: a randomized, controlled trial. Eur J Phys Rehabil Med 2012; 48 (4): 561-567.

8. Berkoff DJ, Miller LE, Block JE. Clinical utility of ultrasound guidance for intra-articular knee injections: a review. Clin Interv Aging 2012; 7: 89-95.

9. Sibbitt WL Jr, Kettwich LG, Band PA: Does ultrasound guidance improve the outcomes of arthrocentesis and corticosteroid injection of the knee? Scand J Rheumatol 2012; 41 (1): 66-72.

10. Kokavec M (Eds). Vybrané kapitoly z detskej ortopédie. Martin; Osveta, 2003, 1-478.

11. Iagnocco A, Perricone $\mathbf{C}$, Scirocco $\mathbf{C}$ et al. The interobserver reliability of ultrasound in knee osteoarthritis. Rheumatology (Oxford) 2012; 51 (11): 2013-2019.

12. Wiler JL, Costantino TG, Filipone L, Satz W. Comparison of ultrasound-guided and standard landmark techniques for knee arthrocentesis. J Emerg Med 2010; 39 (1): 76-82.

13. Ohashi S, Ohnishi I, Matsumoto T et al. Measurement of articular cartilage thickness using a three-dimensional image reconstructed from B-mode ultrasonography mechanical scans feasibility study by comparison with MRI-derived data. Ultrasound Med Biol 2012; 38 (3): 402-411.

14. Niu HJ, Wang Q, Wang YX, Li DY, Fan YB, Chen WF. Ultrasonic reflection coefficient and surface roughness index of OA articular cartilage: relation to pathological assessment. BMC Musculoskelet Disord 2012; 10; 13-34.

15. Eşen S, Akarırmak U, Aydın FY, Unalan H. Clinical evaluation during the acute exacerbation of knee osteoarthritis: the impact of diagnostic ultrasonography. Rheumatol Int 2012 May 5. Epub ahead of print.

Received December 4, 2012. Accepted October 27, 2013. 